\title{
MRI negative myelopathy post mild SARS-CoV-2 infection: vasculopathy or inflammatory myelitis?
}

\author{
Rory M. C. Abrams ${ }^{1} \cdot$ Farinaz Safavi $^{2} \cdot$ Stanley Tuhrim ${ }^{1} \cdot$ Allison Navis $^{1} \cdot$ Jeremy Steinberger $^{3} \cdot$ Susan C. Shin ${ }^{1}$ (I)
}

Received: 12 January 2021 / Revised: 16 April 2021 / Accepted: 10 May 2021 / Published online: 8 June 2021

(c) Journal of NeuroVirology, Inc. 2021

\begin{abstract}
Since the onset of the COVID-19 pandemic, there have been rare reports of spinal cord pathology diagnosed as inflammatory myelopathy and suspected spinal cord ischemia after SARS-CoV-2 infection. Herein, we report five cases of clinical myelopathy and myeloradiculopathy in the setting of post-COVID-19 disease, which were all radiographically negative. Unlike prior reports which typically characterized hospitalized patients with severe COVID-19 disease and critical illness, these patients typically had asymptomatic or mild-moderate COVID-19 disease and lacked radiologic evidence of structural spinal cord abnormality. This case series highlights that COVID-19 associated myelopathy is not rare, requires a high degree of clinical suspicion as imaging markers may be negative, and raises several possible pathophysiologic mechanisms.
\end{abstract}

Keywords Inflammatory myelopathy $\cdot$ Ischemic myelopathy $\cdot$ Transverse myelopathy syndrome $\cdot$ Long Haul Covid $\cdot$ Post Acute Covid-19 Syndrome

\section{Introduction}

Since the onset of the COVID-19 pandemic, there have been increasing reports of neurologic complications including Guillain-Barré syndrome (GBS), meningitis, ischemic stroke, and CNS vasculitis associated with SARS-CoV-2 infection (Ellul et al. 2020; Guidon and Amato 2020; Abrams et al. 2020). There have also been rare reports of myelopathies and suspected spinal cord ischemia typically described in patients hospitalized for moderate-to-severe COVID-19 disease (Zachariadis et al. 2020; Zoghi et al. 2020; Sotoca and Rodríguez-Álvarez 2020; Munz et al. 2020).

However, the potential mechanisms of neurological complications following COVID-19 are not well understood. SARS-CoV-2 infection may affect the central and peripheral

Susan C. Shin

Susan.Shin@mssm.edu

1 Department of Neurology, Icahn School of Medicine At Mount Sinai, 1468 Madison Avenue, New York, NY 10029, USA

2 Section of Infections of Nervous System, Division of Neuroimmunology and Neurovirology NINDS, NIH, Bethesda, Maryland, USA

3 Department of Neurosurgery Icahn School of Medicine At Mount Sinai, 1468 Madison Avenue, New York, NY 10029, USA nervous system through the following three mechanisms: (1) systemic activation of innate immunity following moderate-to-severe infection, leading to endothelial damage and neuronal dysfunction (Quinaglia et al. 2021); (2) enhanced adaptive immunity resulting in an antibody-mediated autoinflammatory process analogous to molecular mimicry (Nath and Smith 2021); and (3) rare direct viral invasion of SARS-CoV-2 to CNS tissues inducing infectious encephalitis (Moriguchi et al. 2020).

During the first year of the pandemic, it has become increasingly apparent, that the spectrum of neurological complications can happen in patients who experienced asymptomatic or only mild COVID-19 disease (Nath 2020). Herein, we report five cases of clinical myelopathy in the setting of post-COVID-19 disease, which were all radiographically negative, which poses additional clinical obstacles to establishing a timely diagnosis. Additionally, these factors raise additional questions about mechanisms of neurological complications attributable to COVID-19.

\section{Case series}

\section{Case 1}

A 69-year-old male presented with progressive leg weakness, numbness, urinary retention, and constipation for 2 months. Examination revealed flaccid paraplegia, a T8 
pinprick sensory level and brisk patellar reflexes. MRI C/T/L spine with contrast, at presentation and a month later, did not reveal spinal cord structural changes or signal abnormalities. Nerve conduction studies (NCS) and electromyography testing (EMG) showed mild denervation at the L3-4 myotomes, but the profound lack of activation was attributed to spinal cord dysfunction. Extensive myeloradiculopathy work up showed strong positive serum SARS-CoV-2 antibody. The patient had no recollection of a preceding respiratory or viral syndrome. Empiric treatment with of IV methylprednisolone $1 \mathrm{~g} /$ day and IV immunoglobulin for 5 days each did not improve symptoms.

\section{Case 2}

A 64-year-old male experienced acute onset of burning paresthesias in the bilateral lower extremities, followed by progressive weakness, numbness, and gait imbalance. He reported an electric-shock sensation down his spine with neck flexion. Examination revealed a mid-thoracic pinprick level, increased patellar reflexes, hip flexion weakness, and difficulty in tandem gait. MRI C/T/L spine did not reveal any cord signal change. NCS/EMG was normal. Myelopathy serologies were negative except for a strong positive SARSCoV-2 antibody. He endorsed prior high-risk COVID-19 exposure followed by a viral syndrome 4 months prior. Improvement in the electric sensation occurred after a course of IV methylprednisolone; however, motor and gait dysfunction persisted.

\section{Case 3}

A 64-year-old female was awakened from sleep by left leg muscle cramps and endorsed weakness and numbness. Examination revealed scissoring gait with diffuse hyperreflexia, Hoffman's signs in the upper extremities, and left lower extremity clonus, upgoing toes, weakness, ataxia, and reduced pinprick sensation without sensory level was present. MRI C/T/L spine did not reveal any abnormalities. EMG showed mild denervation in the left greater than right L5-S1 myotomes. Myelopathy serologies were negative. History was notable for a mild viral syndrome 4 months prior with positive antibodies 2 months after the viral syndrome.

\section{Case 4}

A 58-year-old female experienced acute onset of severe cramping pain in both lower extremities followed by leg weakness and gait imbalance. She had been hospitalized a week prior following treatment with steroids, remdesivir, and apixiban for COVID-19 pneumonia. Examination revealed bilateral hip flexor weakness, a T6 pinprick level, brisk patellar and Achilles reflexes, and broad-based gait. MRI C/T/L spine with contrast was unremarkable. EMG/ NCS was normal. Pain improved following IV methylprednisolone, but motor and gait dysfunction persisted.

\section{Case 5}

A 63-year-old male presented with subacute onset of gait dysfunction and left leg ataxia. Examination revealed pes cavus deformities, brisk patellar and Achilles reflexes, left upgoing toes, and spastic "scissoring" gait with preserved motor strength. One month prior he experienced a viral syndrome of fever and anosmia with confirmed SARS-CoV-2 nasopharyngeal PCR. MRI C/T/L spine was unrevealing. NCS/EMG revealed mild denervation in the $\mathrm{S} 1$ myotomes, normal motor unit recruitment pattern but diminished activation attributed to central dysfunction. Genetic testing revealed a pathogenic mutation for autosomal dominant hereditary spastic paraplegia (HSP) 10 . However, the patient adamantly denied any neurologic symptoms prior to COVID-19 disease or family history of HSP (Table 1).

\section{Discussion}

To our knowledge, this represents the largest series to date of post-COVID-19 associated myelopathy and myeloradiculopathy and uniquely identifies a phenotype affecting patients with only mild or asymptomatic SARS-CoV-2 infection. In all five cases, the patients presented with acute or subacute onset of lower extremity paresis, sensory changes, and gait dysfunction. Unlike GBS, the brisk deep tendon reflexes, spinal sensory levels, and normal distal NCS suggested upper motor neuron dysfunction which generally localized to the thoracic and lumbar cord. Unlike prior case reports which typically characterized hospitalized patients with severe COVID-19 disease, critical illness, and often radiographically apparent brain or spine signal change, this series of patients had asymptomatic or mild-moderate COVID19 disease and lacked radiologic evidence of spinal cord abnormality. Radiologic findings in acute inflammatory myelopathies may be absent in up to $40 \%$ of cases (Scotti and Gerevini (2001)), however, the clinical severity of these myelopathies was out of proportion to either the radiologic findings or the severity of the COVID-19 disease suggesting a novel phenotype for clinicians to be aware of.

This series also highlights the prognostic challenge of predicting individuals who might develop severe neurological complications including spinal cord dysfunction. In most of these cases, there were no significant underlying medical issues that might predispose these patients to developing myelopathy or neuropathy. Only case 5 had any indication of a pre-existing condition (HSP) which could have contributed 


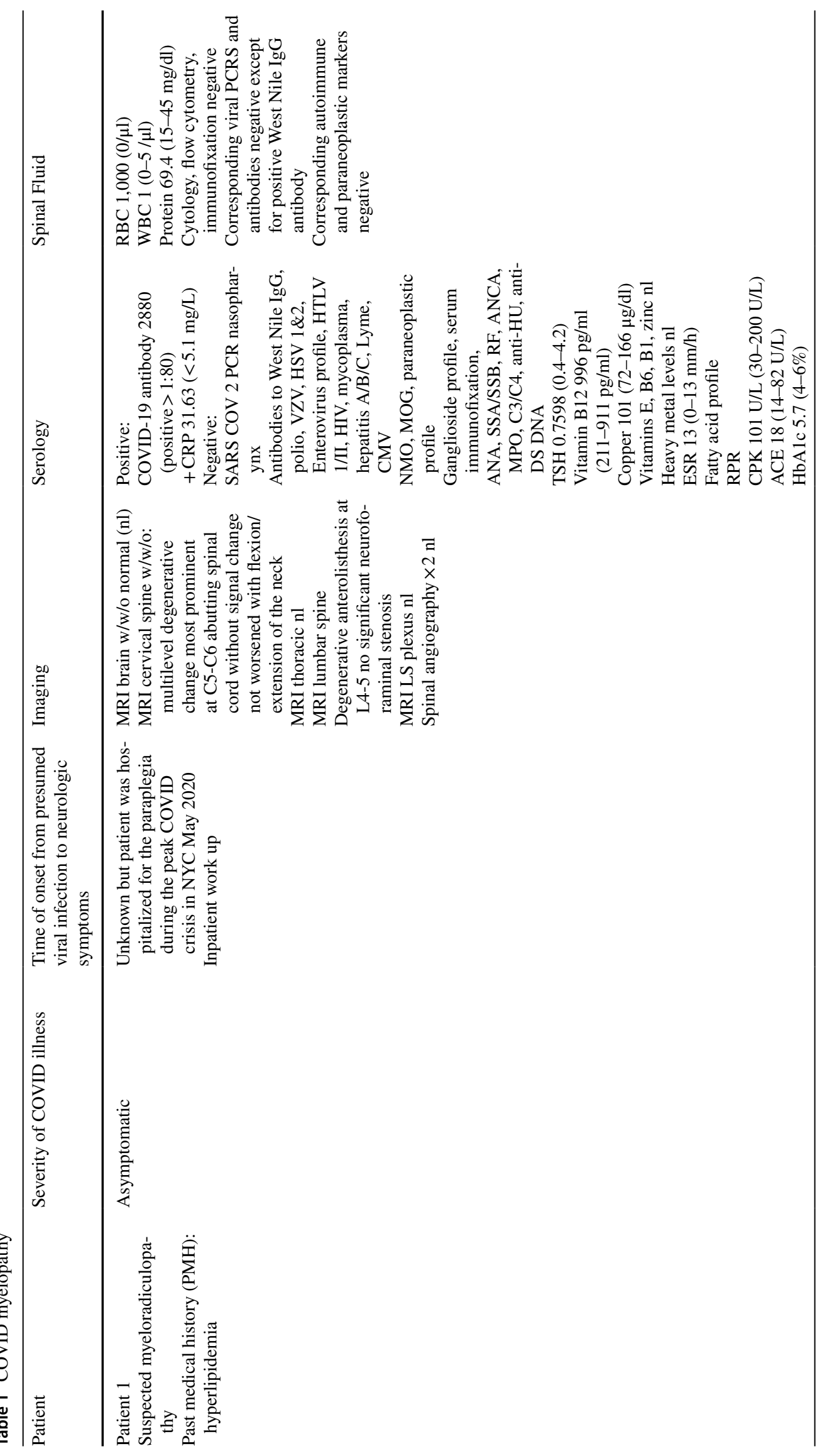




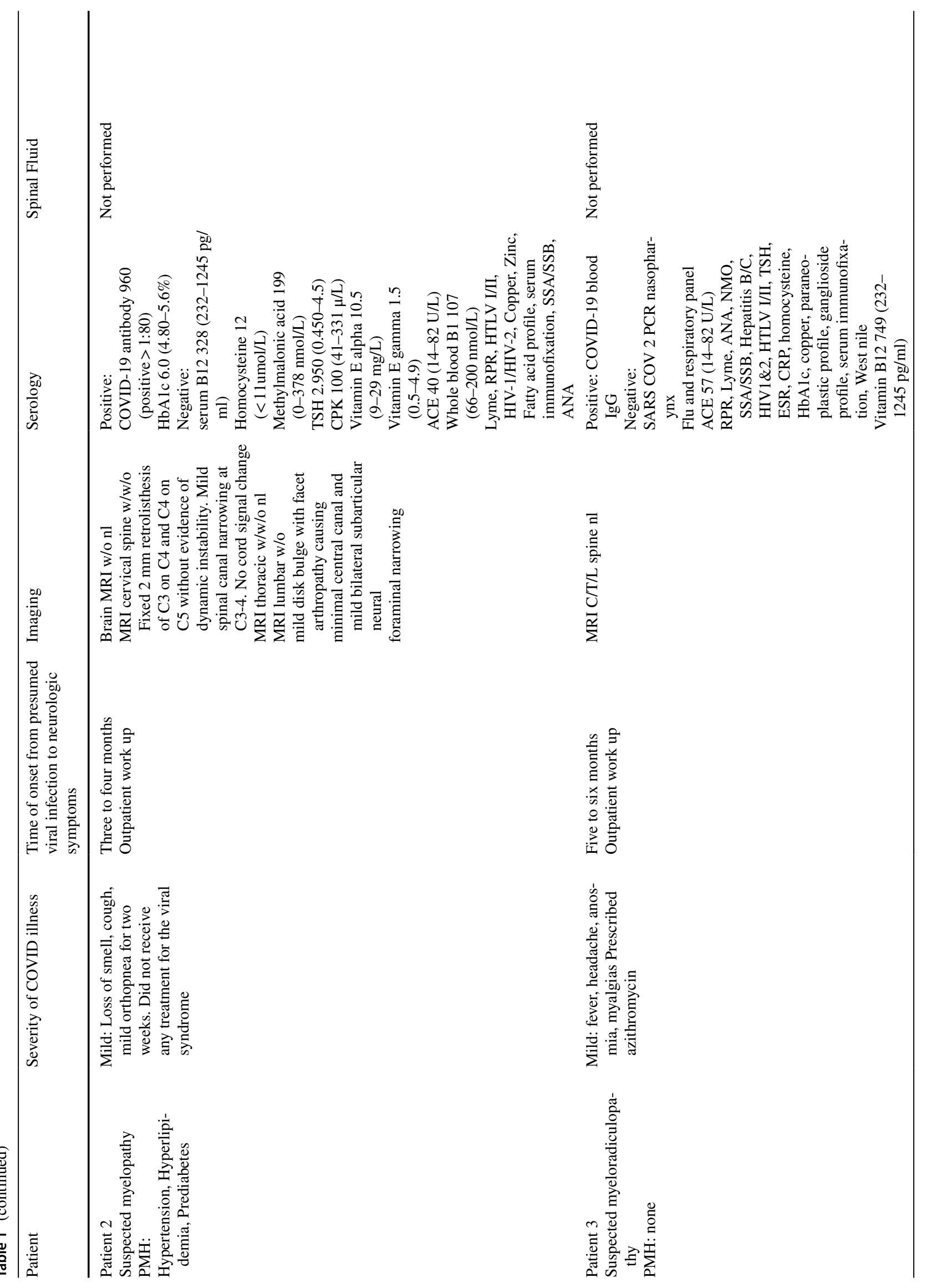




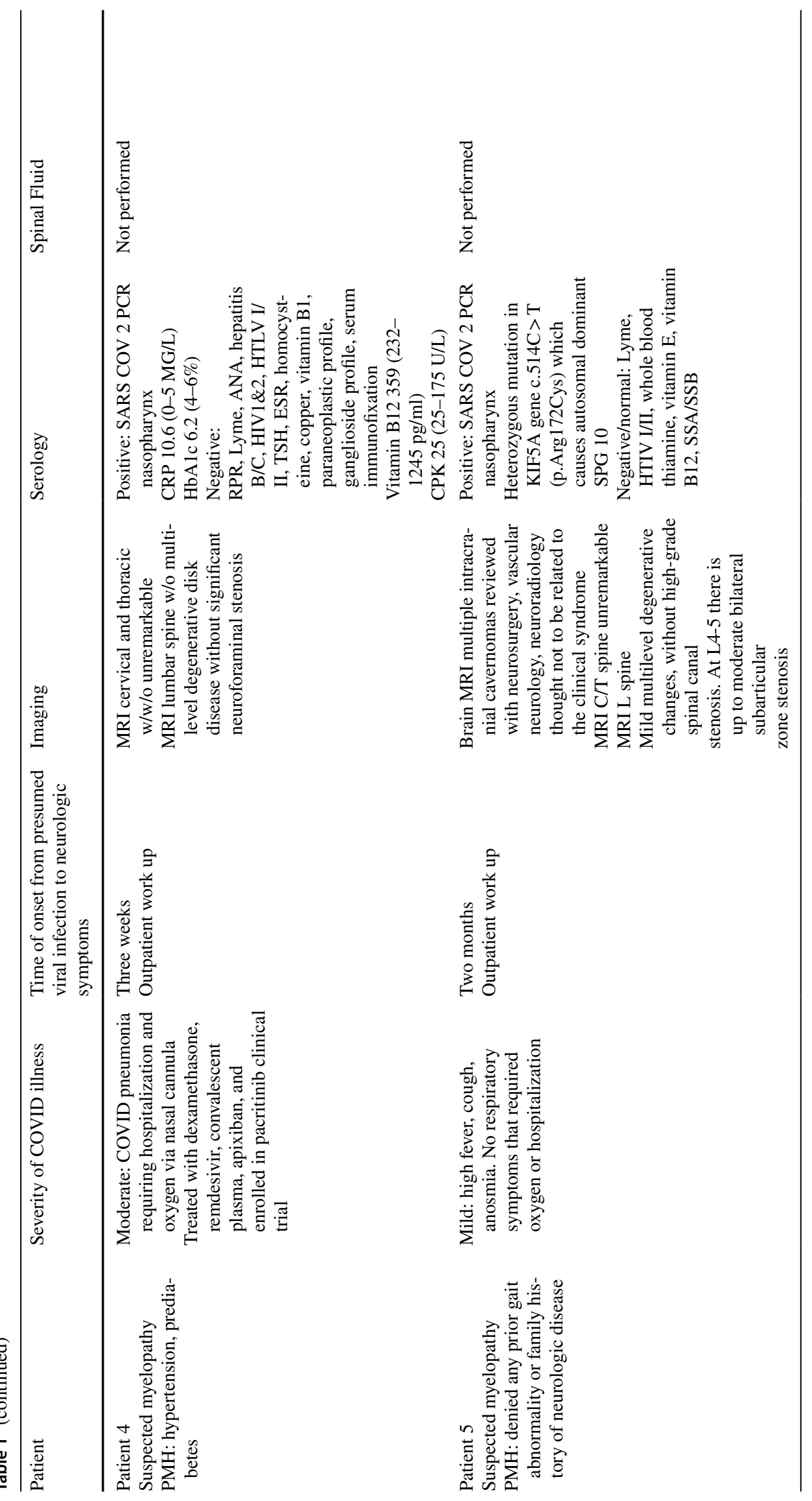


to the clinical findings; this underlying genetic susceptibility may have been amplified by the preceding SARS-CoV-2 infection. However, in the other cases the preceding viral infection seems to be the sole explanation identified for the onset of a myelopathy. The specific pathophysiology in these cases remains unclear.

Other reports of inflammatory myelitis in the postCOVID-19 setting were also frequently delayed by weeks following the acute infection (Zachariadis et al. 2020; Zoghi et al. 2020; Munz et al. 2020). One hypothesis is that the evolution of IgM and IgG SARS-CoV-2 serologies may generate an autoinflammatory state (Ellul et al. 2020) or be due to a delayed T-cell response (Guidon and Amato 2020). To counter the inflammatory response, empiric treatments for inflammatory myelopathies such as steroids have been employed (Sotoca and Rodríguez-Álvarez 2020; Munz et al. 2020). Additionally, all patients herein were older than 55 years old, which is possibly indicative that aging might contribute to the development of inflammatory myelopathy with atypical radiological features due to an imbalance in immune responses (Fülöp et al. 2019).

Unfortunately, like some other authors (Zachariadis et al. 2020; Zoghi et al. 2020), our cases experienced minimal clinical recovery following standard immunotherapies like high-dose steroids. This may indicate a different etiology of the myelopathies in these patients. SARS-CoV-2 infection has been shown to cause both microhemorrhages and small vessel micro-thromboses through endothelial damage, coagulopathies, and antiphospholipid antibody-like syndrome (McFadyen et al. 2020; Salamanna et al. 2019 Jul 3; Barrantes 2021). Small vessel spinal cord strokes may explain the lack of apparent radiologic findings, lack of response to immunotherapeutic agents, and less recovery.

This case series highlights that COVID-19 associated myelopathy is less rare than previously described, particularly given the potential for subtle and delayed neurologic manifestations. COVID-19 associated myelopathy requires a high degree of clinical suspicion as imaging markers may be negative even with severe neurologic presentations. As such, this emphasizes the importance of improving prompt recognition to hopefully implement ameliorating treatments and augment recoveries, but more importantly underscores preventive public health measures which may avoid potentially life-alternating neurologic manifestations.

Acknowledgements This work was supported in part by the Intramural Research Program of the National Institute of Neurological Disorder and Stroke at National Institutes of Health.

\section{References}

Abrams RMC, Kim BD, Markantone DM, Reilly K, Paniz-Mondolfi AE, Gitman MR et al (2020) Severe rapidly progressive GuillainBarré syndrome in the setting of acute COVID-19 disease. J Neurovirol 26(5):797-799

Barrantes FJ (2021) The unfolding palette of COVID-19 multisystemic syndrome and its neurological manifestations. Brain Behav Immun Health 14:100251

Ellul MA, Benjamin L, Singh B, Lant S, Michael BD, Easton A et al (2020) Neurological associations of COVID-19. Lancet Neurol

Fülöp T, Larbi A, Witkowski JM (2019) Human inflammaging. Gerontology 65(5):495-504

Guidon AC, Amato AA (2020) COVID-19 and neuromuscular disorders. Neurology

McFadyen JD, Stevens H, Peter K (2020) The emerging threat of (Micro)thrombosis in COVID-19 and its therapeutic implications. Circ Res 127(4):571-587

Moriguchi T, Harii N, Goto J, Harada D, Sugawara H, Takamino J et al (2020) A first case of meningitis/encephalitis associated with SARS-Coronavirus-2. Int J Infect Dis 94:55-58

Munz M, Wessendorf S, Koretsis G, Tewald F, Baegi R, Krämer S et al (2020) Acute transverse myelitis after COVID-19 pneumonia. J Neurol 267(8):2196-2197

Nath A. Neurologic complications of coronavirus infections. Neurology (2020)May 12;94(19):809-810

Nath A, Smith B (2021) Neurological issues during COVID-19: An overview. Neurosci Lett Jan 18;742:135533

Quinaglia T, Shabani M, Breder I, Silber HA, Lima JAC, Sposito AC (2021) Coronavirus disease-19: The multi-level, multi-faceted vasculopathy. Atherosclerosis 322:39-50

Salamanna F, Maglio M, Landini MP, Fini M. Platelet functions and activities as potential hematologic parameters related to Coronavirus Disease 2019 (Covid-19). Platelets 2020 Jul 3;31(5):627-632

Scotti G, Gerevini S (2001) Diagnosis and differential diagnosis of acute transverse myelopathy. The role of neuroradiological investigations and review of the literature. Neurol Sci Suppl 2:S69-73

Sotoca J, Rodríguez-Álvarez Y (2020) COVID-19-associated acute necrotizing myelitis. Neurol Neuroimmunol Neuroinflamm. 7(5):e803. Published 2020 Jun 10

Zachariadis A, Tulbu A, Strambo D, Dumoulin A, Di Virgilio G (2020) Transverse myelitis related to COVID-19 infection J Neurol 267(12):3459-3461

Zoghi A, Ramezani M, Roozbeh M, Darazam IA, Sahraian MA (2020) A case of possible atypical demyelinating event of the central nervous system following COVID-19. Mult Scler Relat Disord 44:102324

Publisher's Note Springer Nature remains neutral with regard to jurisdictional claims in published maps and institutional affiliations. 